\title{
Information Concealing Based on Outer P-sets
}

\author{
Tan Xiao-dong and Ruan Qun-sheng \\ (1 Hezhou University, Hezhou Guangxi, 542899; 2 Ningde Normal University, \\ Ningde Fujian, 352100) \\ tanxd2004@163.com
}

\begin{abstract}
Outer P-set, a new mathematic method of studying dynamic information, is a part of $P$-set theory. Based on the outer P-set theory, such conceptions have been put forward as principles of $F$-information concealing granulation degree, $F$-information concealing relationship, $F$-information concealing generation, concealing and concealing information recovery and definitions of outer-distance and concealing granulation degree. Their applications have been illustrated through simple examples in application.
\end{abstract}

Keywords: outer P-set, F-information concealing, outer-distance, information recovery

\section{Introduction}

Dynamic traits has been introduced (Shi Kai-quan, 2008) to the limited X-set in order to improve it and P-set, a new set with dynamic traits, has been put forward, which offers a new mathematic approach and module to dynamic information system research. P-set is made up of internal and outer sets and its conceptions, structure, characters and application have been detailed in references [1-6]. In Information system, early warning information is set as $X=\left\{x_{1}, x_{2} \cdots, x_{m}\right\}$ with its property set $\alpha=\left\{\alpha_{1}, \alpha_{2}, \cdots, \alpha_{k}\right\}$ and $X$ needs concealing in order to safely effectively deliver $X$ information, where outer P-set theory and its attributes are applied to obtain $X$ concealing data. In other word, supplements of set $\alpha$ are needed to form a new set $\alpha^{\bar{F}}$ in order to meet the $\alpha^{\bar{F}}$ reduction in $X$ and form the concealing $X^{F}$. The concealing information capacity is larger than the non-concealing one with its reduction dependant on concealing particle degree, the bigger the particle degree and the larger the concealing information capacity. According to the dynamic traits of outer P-set, constant supplements of new attributes to $X$ 's $\alpha$ can be made and $\alpha$ generates $\left\{\alpha_{i}^{\bar{F}}\right\}_{i=1,2, \cdots, n}$, meeting $\alpha \supseteq \alpha_{1}^{\bar{F}} \supseteq \alpha_{2}^{\bar{F}} \supseteq \cdots \supseteq \alpha_{n-1}^{\bar{F}} \supseteq \alpha_{n}^{\bar{F}}$, and $X$ generates $\left\{X_{i}^{F}\right\}_{i=1,2, \cdots, n}$, with $X_{i}^{F}$ set $\alpha_{i}^{\bar{F}}$, meeting $X_{n}^{F} \supseteq X_{n-1}^{F} \supseteq \cdots \supseteq X_{2}^{F} \supseteq X_{1}^{F} \supseteq X$.X here can be viewed as $X_{n}^{F}$ 's concealing set. To meet demands of maritime information safety, the required concealing information chosen from $X_{n}^{F}$ concealing set is delivered from sender to the receiver. Information concealing-restore computing is needed to accomplish information recovery by the receiver when received. Concealing-restore computing is a converse one of concealing operation, whose technical theory will be detailed in this essay. In order to solve above problem of information concealing and restore in fishing-boat warning, such conceptions have been first put forward as principles of $F$-information concealing granulation degree, n-rank $F$-information concealing relationship, $F$ information concealing generation, light concealing and concealing information recovery and definitions of outer-distance, concealing granulation degree, relative reasoning degree and light concealing deviation degree. The theory and approach to the safety of marine warning information sending and receiving have been then analyzed and illustrated with examples. Internal As knowledge preparation, P-set structure has been introduced in the 
first part of this essay in order to keep the integrity of the analysis.

\section{Structure of Outer P-set ${ }^{[7-11]}$}

Definition 2.1 $\mathrm{U}$ is defined as limited element domain, $\mathrm{V}$ is the limited character domain and X U's limited non-empty set. $F=\left\{f_{1}, f_{2}, \cdots, f_{m}\right\}$ and $\bar{F}=\left\{\bar{f}_{1}, \bar{f}_{2}, \cdots, \bar{f}_{n}\right\}$ are defined as the element transferable group, with $f \in F$ and $\bar{F}=\left\{\bar{f}_{1}, \bar{f}_{2}, \cdots, \bar{f}_{n}\right\}$ as element transfers $^{[1-3]}$.

Definition 2.2 Given Limited common set $(x)=\left\{x_{1}, x_{2}, \cdots, x_{m}\right\} \subset U, \quad \alpha=\left\{\alpha_{1}, \alpha_{2}, \cdots\right.$,

$\left.\alpha_{k}\right\} \subset V$ is defined as the character set of $(x)$ and $(x)^{F}$ is the generated outer P-set of $X$, in short, $(x)^{F}$ is outer P-set, and

$(x)^{F}=(x)+(x)^{+}$

Where $(x)^{F}$ is called $F$ - element add set of $X$, so $(x)^{F}=\{x \mid x \in X, f(x)=u \in X, f \in F\}$, with character set $\alpha^{\bar{F}}$ of $(x)^{F}$ satisfied by

$\alpha^{F}=\alpha-\left\{\alpha^{\prime} \mid f^{-}(\beta)=\alpha^{\prime} \notin \alpha, f \notin F\right\}$

In definition 2.2, if members of $\alpha$ are omitted, which turn $\alpha$ into $\alpha_{1}^{\bar{F}}$ and generating $(x)_{1}^{F}$, satisfied by $\alpha \supseteq \alpha_{1}^{\bar{F}}, \quad(x)_{1}^{F} \supset(x)$. If more characters are omitted from $\alpha$,turning $\alpha$ into $\alpha_{2}^{\bar{F}}$ and generating $(x)_{2}^{F}$, satisfied by $\alpha_{1}^{\bar{F}} \supseteq \alpha_{2}^{\bar{F}}$, so $(x)_{2}^{F} \supset(x)_{1}^{F}$. If minuses of $n$ times are made, turning $\alpha$ into $\alpha_{n}^{\bar{F}}$, and generating $(x)_{n}^{F}$, satisfied by $\alpha_{n-1}^{\bar{F}} \supseteq \alpha_{n}^{\bar{F}}$ and $(x)_{n-1}^{F} \subseteq(x)_{n}^{F}$ 。

Proposition 1. (x) 's outer P-set serial of $\left\{(x)_{i}^{F}\right\}_{i=1,2, \cdots, n}$ meets Expression 3 as:

$(x)_{n}^{\bar{F}} \supseteq(x)_{n-1}^{\bar{F}} \supseteq \cdots \supseteq(x)_{2}^{\bar{F}} \supseteq(x)_{1}^{\bar{F}} \supseteq(x)$

Proposition2. Character set serial $\left\{\alpha_{i}^{\bar{F}}\right\}_{i=1,2, \cdots, n}$ of $(x)$ 's outer P-set serial of $\left\{(x)_{i}^{F}\right\}_{i=1,2, \cdots, n}$ meets Expression 4 as:

$\alpha \supseteq \alpha_{1}^{\bar{F}} \supseteq \alpha_{2}^{\bar{F}} \supseteq \cdots \supseteq \alpha_{n-1}^{\bar{F}} \supseteq \alpha_{n}^{\bar{F}}$

\section{3. $F$ - Information Generation and Relation Principle}

Definition 3.1 If $(x)=\left\{x_{1}, x_{2}, \cdots, x_{p}\right\} \subset U$ is defined as an information set of $U$ and $x_{i} \in(x)$ as the information unit of $(x)$ and $i=1,2, \cdots, p$ with $(x)$ having the limited character $\alpha$ and satisfied by

$\alpha=\left\{\alpha_{1}, \alpha_{2}, \cdots, \alpha_{k}\right\}$

Definition 3.2 If $(x)^{F} \subset U$ is defined as a $F$ - information set of $(x)$ and satisfied by

$(x)^{\mathrm{F}}=\left\{x_{1}, x_{2}, \cdots, x_{q}\right\}$

So $(x)^{F}$ has a limited character set $\alpha^{\bar{F}}$ and is satisfied by Expression 7 .

$\alpha^{\bar{F}}=\alpha-\left\{\beta_{i} \mid f^{-}\left(\alpha_{i}\right)=\beta_{i} \notin \alpha, f^{-} \notin F\right\}$

Where $p \leq q ; p, q \in \mathbf{N}^{+}$

Definition 3.3 If $(x)_{n}^{F}$ is defined as $n$-rank outer inducing information set of $(x),(x)_{n}^{F}$ as $F$ - information of $(x)_{n-1}^{F}$ with satisfaction of

$\alpha_{n}^{\bar{F}}=\alpha_{n-1}^{\bar{F}}-\left\{\beta_{i} \mid f^{-}\left(\alpha_{i}\right)=\beta_{i} \notin \alpha_{n-1}^{\bar{F}}, f^{-} \notin F\right\}$

Where $(x)_{0}^{F}=(x)$. 
Definition 3.4 If $C R_{n}^{F}$ is defined as ( $x$ )'s relative outer inductive degree of outerinducing information set and satisfied by $C R_{n}^{F}=\operatorname{card}((x)) / \operatorname{card}\left((x)_{n}^{F}\right)$

The card in expression 9 is the same as cardinal number。

From the four definitions above, two propositions will be obtained as:

Proposition 3 In information set of $(x)$, the smaller relative outer-inducing information degree of outer-inducing information $(x)_{n}^{F}$ is, the higher information concealing degree is and true is the converse.

Proposition 4 The information $(x)_{n}^{F}$ of relative outer-inducing information degree $C R_{n}^{F}<1$ is a $F$ - concealing information of $(x)$ set and true is the converse.

Principle 1 (Principle of $F$ - information concealing particle) If $\sigma_{i}$ is the concealing particle degree of $(x)_{i}^{F}$ in $F$ - information set $\left\{(x)_{i}^{F} \mid i=1,2, \cdots, n\right\}, \sigma_{i}=$ $\operatorname{card}((x)) / \operatorname{card}\left((x)_{i}^{F}\right)$ with $0<\sigma_{i}<\sigma_{i-1}<\cdots<\sigma_{2}<\sigma_{1}<1$

Proof From expressions (2), (7), (8) and Proposition 1, 2, character set serial $\left\{\alpha_{i}^{\bar{F}} \mid i=1, \quad 2,3, \cdots, n\right\} \quad$ of $\quad F \quad$ - concealing set $\left\{(x)_{i}^{F} \mid i=1,2, \cdots, n\right\} \quad$ satisfying $\alpha \supset \alpha_{1}^{\bar{F}} \supset \alpha_{2}^{\bar{F}} \supset \cdots \supset \alpha_{n-1}^{\bar{F}} \supset \alpha_{n}^{\bar{F}}$ is obtained. If relative outer-inducing degree $C R_{n}^{F}=$ $\operatorname{card}((x)) / \operatorname{card}\left((x)_{n}^{F}\right)$ of $(x)_{i}^{F}$ in Expression 4, then $C R_{n-1}^{F}<C R_{n}^{F}<\cdots<C R_{2}^{F}<C R_{1}^{F}<1$. If $\operatorname{card}(x)_{n}^{F}$ with $\operatorname{card}(x)$ non-negative, then $C R_{n}^{F}>0$. So Expression 10 is tenable.

Deduction 1 If character set $\alpha_{i}^{\bar{F}}$ of $(x)_{i}^{F}$ and $(x)$ with $\alpha$ satisfying $\alpha-\alpha_{i}^{F}=\varnothing$, then $\operatorname{UNI}\left\{(x)_{i}^{F},(x)\right\}$ where $U N I=$ unidentification.

In fact, according to P-set definition and in accordance with Expression 10, if $\alpha-\alpha_{i}^{F}=\varnothing, \operatorname{card}((x)) / \operatorname{card}\left((x)_{i}^{F}\right)=1$ can be deduced when the two character sets are same. It is shown that $(x)_{i}^{F}$ and $(x)$ are two unidentified sets.

Principle 2 (Principle of $k$-rank concealing information relation) If $(x)_{n}^{F}$ is $k$-rank $F$ - information of $(x)$ information, then it satisfies

$(x)_{n}^{F} \supseteq(x)_{n-1}^{F} \supseteq \cdots \supseteq(x)_{1}^{F}$

Proof According to Expressions 5 to 9, character set $\alpha_{n}^{\bar{F}}, \alpha_{n-1}^{\bar{F}}, \alpha_{1}^{\bar{F}}$ of $(x)_{n}^{F},(x)_{n-1}^{F},(x)_{1}^{F}$ can be obtained satisfying $\alpha_{1}^{F} \subseteq \alpha_{n-1}^{F} \subseteq \alpha_{n}^{F}$. According to Definition 2.4 and Principle 1, $(x)_{n}^{F} \supseteq(x)_{n-1}^{F} \supseteq \cdots \supseteq(x)_{1}^{F}$ is obtainable.

Principle 3 ( $F$ - concealing information generation principle) If information $(x)$ 's character set $\alpha$ of $(x)^{F}$ concealing information satisfies

$\alpha^{\bar{F}}=\alpha-\left\{\alpha_{i} \mid f^{-}\left(\alpha_{i}\right)=\beta_{i} \notin \alpha^{\bar{F}}, f^{-} \notin F\right\}$

in $(x)$, if part of information unit is added, then $(x)$-based $F$ - concealing information of $(x)^{\bar{F}}$ is generated.

Proof Expression 13 equals $\alpha^{\bar{F}} \subseteq \alpha, \operatorname{card}\left(\alpha^{\bar{F}}\right) \geq \operatorname{card}(a)$. From expressions of (5), (6), (7), $(x)^{F}$ can be inferred to be generated by the deleted information unit and, from expression, $(x)^{F}$ can be inferred to be $(x)$ 's $F$ - concealing information. 


\section{Information Concealing and Recovery Theory}

Definition 4.1 If $\psi^{F}$ is defined as $(x)^{F}$,s light concealing deviation degree and

$$
\psi^{F}=\|(x)^{F} \square / \square(x) \square
$$

Where $\square(x)^{F} \square=\left((x)_{1}^{2}+(x)_{2}^{2}+\cdots+(x)_{p}^{2}\right)^{1 / 2}$ is 2-norm of vector $(x)^{F}=\left((x)_{1},(x)_{2}, \cdots,(x)_{p}\right)^{\mathrm{T}}$ and $(x)^{F}=\left((x)_{1},(x)_{2}, \cdots,(x)_{p}\right)^{\mathrm{T}}$ is the vector generated by characteristic value $x_{i} \in(x)^{F}\left(x_{i}\right.$ value) ${ }^{[12]}$, here: $i=1,2, \cdots, p$.

Definition 4.2 If $L_{i, j}^{F}$ is the outer-distance of concealing information $(x)_{i}^{F}$ and

$L_{i, j}^{F}=\left|\psi_{i}^{F}-\psi_{j}^{F}\right|$

If $\psi_{i}^{F}$ and $\psi_{j}^{F}$ are respectively light concealing deviation degrees of $(x)_{i}^{F}$ and $(x)_{j}^{F}$ at time of $t_{i}$ and $t_{j}$.

Principle 4 (principle of information concealing) If concealing granulation degree $\sigma<1$ is given with $(x)^{F}$ the character of $\sigma$, then $(x)^{F}$ is a concealing of $(x)$ with $(x)$ embedded in $(x)^{F}$.

Proof According to Expression 9, $\sigma=\operatorname{card}((x)) / \operatorname{card}\left((x)_{i}{ }^{F}\right)<1,(x)_{i}{ }^{F}$ is a concealing one of $F$ - information generated by $(x)$. From Expression $12,(x)^{F} \supseteq(x)$ can be inferred, that is, the numbers of $(x)_{i}{ }^{F}$ information unit are more than $(x)$. So $(x)^{F}$ is a concealing of $(x)$ with $(x)$ embedded in $(x)_{i}{ }^{F}$. So Principle 4 can be illustrated. Its further expression is illustrated as Figure 1.

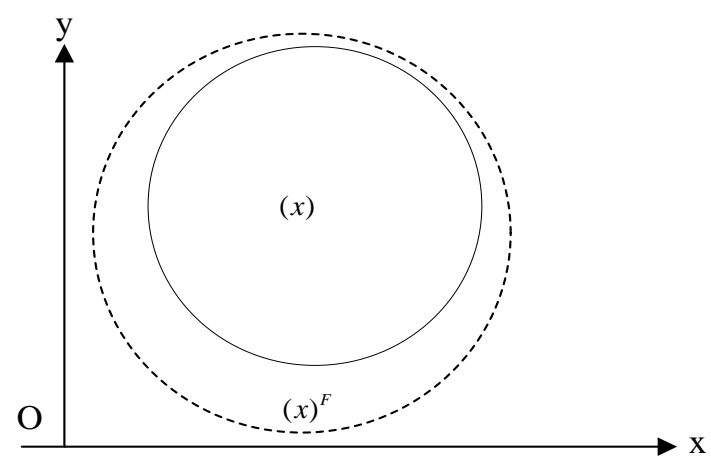

Figure 1. Information Unit Area of $(x)$ Shown by Solid Line Circle, Concealing Information of $(x)^{\bar{F}}$ by Dotted Line Circle, $(x)$ Embedded in $(x)^{F}$, $x, y$ Shows the Vertical and Horizontal Coordinates of $(x)$ and $(x)^{F}$

Principle 5 (Principle of concealing information recovery) A sufficient and necessary condition for $(x)_{j}^{F}$ recovered from concealing information $(x)_{i}^{F}$ is $\psi^{F}$, with $L_{i, j}^{F}$ satisfying $L_{i, j}^{F}=0, \psi^{F}=0$

Proof (i) if $(x)_{j}^{F}$ is recovered into $(x)_{i}^{F}$, or $(x)_{i}^{F}=(x)_{j}^{F}$, according to Expressions 10 and $11, \sigma_{i}=\sigma_{j}$ can be obtained with $(x)_{j}^{F}$ and $(x)_{i}^{\bar{F}}$ unidentified. From Expression 14 and 15 , if $L_{i, j}^{F}=0, \psi^{F}=0$, Expression 16 is tenable.

(ii) From Express 15, if $c=0, \psi^{F}=0$, then $L_{i, j}^{F}=\left|\sigma_{i}^{F}-\sigma_{j}^{F}\right|=0$ an $\mathrm{d} \quad \sigma_{i}^{F}=\sigma_{j}^{F}$. According to Expressions 14 and 15, if $\|(x)_{i}{ }^{F} \square=\square(x)_{j}{ }^{F} \square$, then $(x)_{i}{ }^{F}=(x)_{j}{ }^{F}$, where 
$\left\|(x)_{i}{ }^{F}\right\|$ and $\left\|(x)_{j}{ }^{F}\right\|$ are respectively 2-norm of vector $(x)_{i}{ }^{F}$ and $(x)_{j}{ }^{F}$. They are vectors generated by characteristic value sets of $(x)_{j}^{F}$ and $(x)_{i}^{F}$. So $(x)_{j}^{\bar{F}}=(x)_{i}^{\bar{F}}$ and concealing information $(x)_{j}^{F}$ can be recovered into $(x)_{i}^{F}$.

Information Identification Criterion The $(x)^{F}$, concealing information, is generated by $(x)$. If $L_{i, j}^{F}$, outer-distance of concealing information, is satisfying:

$L_{i, j}^{F} \geq \ell$

$(x)^{F}$ is qualified concealing information based on $(x)$. notes: $\ell$ is a threshold value about information concealing.

\section{Applications of Theory}

The data of this section is acquired from typhoon warning system(Notes: the data in application has been abstracted.). Given $(x)=\left\{x_{1}, x_{2}, \cdots, x_{9}\right\}$ the information set for transformation, where the data is abstracted as table 1 with its character variable set $\alpha=\left\{\alpha_{1}, \alpha_{2}, \alpha_{3}, \ldots, \alpha_{q}\right\}$.

Table 1. The Sample Data $(x)$ of the Warning Information before Concealing

\begin{tabular}{cccccccccc}
\hline$(x)$ & $x_{1}$ & $x_{2}$ & $x_{3}$ & $x_{4}$ & $x_{5}$ & $x_{6}$ & $x_{7}$ & $x_{8}$ & $x_{9}$ \\
\hline$y$ & 119.68 & 119.36 & 119.07 & 119.28 & 119.62 & 119.08 & 119.76 & 119.24 & 119.69 \\
\hline
\end{tabular}

According to theories, principles and definitions of outer P-set, if information unit in character variable set $\alpha$ of $(x)$ is reduced, so $(x)=\left\{x_{1}, x_{2}, \cdots, x_{m}\right\}, m<n$ and $(x)$ is concealing as $(x)^{F}$, with its character set $\alpha^{F}=\left\{\alpha_{1}, \alpha_{2}, \alpha_{3}, \ldots, \alpha_{p}\right\}, q>p$. The abstracted data of $F$ - information $(x)^{F}$ is illustrated as table 2 .

Table 2. The Outward-Recursion Data ${ }^{(x)_{i}{ }^{F}}$ of ${ }^{(x)}$ after Concealing

\begin{tabular}{ccccccc}
\hline$(x)_{1}^{F}$ & $x_{1}$ & $x_{2}$ & $x_{3}$ & $\ldots$ & $x_{15}$ & $x_{16}$ \\
\hline$y_{1}^{F}$ & 119.34 & 119.86 & 119.26 & $\cdots$ & 119.38 & 119.54 \\
\hline
\end{tabular}

According to Expressions 1-9, data of Figure 2 can be generated by that of table 1 . According to principle 4 and relative definitions, information in Figure 1 is included in Figure 1, that is, $(x)^{\bar{F}}$ embedded in $(x)$. From Principle 1, Definition 3.1 and Definition 3.2, the conceptions as concealing granulation degree, concealing deviation degree and concealing outer distance can be used to judge information concealing degree and their values can be set in accordance with applications. Concealing operations on $k$-rank $F$ information can be repeatedly made until the required concealing information has been generated. According to Principle 5 and 2, concealing information $(x)^{F}$ in table 2 can be recovered into $(x)$. From Deduction 1 and 2, it is inferred that information can be utmost recovered when $(x)^{F}$ and $(x)$ satisfy $U N I\left((x),(x)^{F}\right)$ or $L_{i, j}^{F}=0$. We can evaluate the quality of $(x)_{i}{ }^{F}$, According to the information concealing criterion in this paper.

\section{Conclusion}

Such conceptions, based on the outer P-set theory, have been put forward as principles of $F$-information concealing granulation degree, $F$-information concealing relationship, $F$-information concealing generation, concealing and concealing information recovery 
and definitions of outer-distance and concealing granulation degree so as to offer theoretical support for the safe transformation of important information as text and images. In practices, it has been founded that the theory and approaches of the P-set-based information concealing relationship and generation have a good reference to both the information safety operation and the system information safety field. The theories in this paper are given are good for information camouflage.

\section{Acknowledgement}

This work is supported by the University Science Foundation of Guang Xi Province P. R China under Grant No. ZD2014129, which is approved and initiated in 2014. The authors are also grateful to the valuable comments and suggestions of the reviewers.

\section{References}

[1] S. Kaiquan, "P-sets and its applications[J]", An International Journal Advances in Systems Science and Applications, vol. 9, no. 2, (2009), pp. 209-219.

[2] S. Kaiquan. "P-sets [J]", Journal of Shandong University: Science Journal, vol. 43, no. 11, (2008), pp. 77-84.

[3] L. Yu-ying, R. Qun-sheng, Z. Shi-liang, "Identification and recovery of inward-recursion information [J]", Journal of Shandong University: Science Journal, vol. 46, no. 6, (2011), pp. 121-126.

[4] S. Kaiquan, "inner P-sets [J]", Journal of Shandong University: Science Journal, vol. 47, no. 11, (2012), pp. 98-109.

[5] L. Hongkang, F. Chengxian, "The dual form of P-reasoning and identification of unknown attribute[J]", International Journal of Digital Content Technology and its Applications, vol. 6, no. 1, (2012), pp. 121-131.

[6] S. Kai-quan, T. Ji-hua, Z. Ling, "Intelligent fusion of inverse packet information and recessive transmission of information's intelligent hiding [J]", Systems Engineering and Electronics, vol. 35, no. 3, (2015), pp. 117-124.

[7] L. Hongkang, L. Yuying, "P-sets and its P-separation theorems[J]", An International Journal Advances in Systems Science and Applications, vol.10, no. 2, (2010), pp. 209-215.

[8] H. Shunliang, W. Wei, G. Dianyou, "P-sets and its internal P-memory characteristics [J]", An International Journal Advances in Systems Science and Applications, vol. 10, no. 2, (2010), pp. 216-222.

[9] L. Yuying, Z. Li, S. Kaiquan, "Generation and recovery of compressed data and redundant data[J]". Quantitative Logic and Soft Computing, vol. 2, no.1, (2010), pp. 661-671.

[10] L. Yuying, R. Qunsheng, "Inner-recursion information identification-recovery [J]",Journal of Shandong University:Science Journal, vol. 46, no. 6, (2013), pp. 71-75.

[11] S. Kaiquan, L. Xiuhong, "Concealing information identification and its applications [J]" An International Journal Advances in Systems Science and Applications, vol. 10, no. 2, (2010), pp. 157167.

[12] L. Hongkang, L. Yuying, X. Gou-min, "Dynamic data distance and Geometric identification- recovery of dynamic data [J]", Journal of Shandong University: Science Journal, vol. 48, no. 7, (2014), pp. $134-140$

\section{Authors}
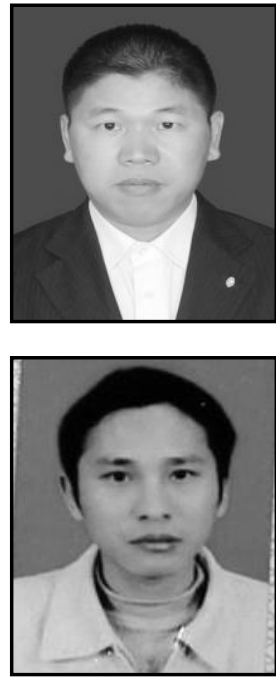

246
Tan Xiao-dong, He was born in May,1974. He is a lecture in He Zhou University. His research interests are the analysis and design of algorithm, computer network, etc.

Ruan Qun-sheng, He was born on Sep 9, 1979. He is an associated in Ning De University. His research interests are design and analysis of algorithms, computer software and theory, etc. 